\title{
The Incontinentia Pigmenti Genetic Biobank: study design and cohort profile to facilitate research into a rare disease worldwide
}

\author{
Francesca Fusco ${ }^{1}$. Valeria Valente ${ }^{1} \cdot$ Dario Fergola $^{1} \cdot$ Alessandra Pescatore $^{1} \cdot{\text { Maria Brigida } \text { Lioi }^{2}}^{*}$ \\ Matilde Valeria Ursini ${ }^{1}$
}

Received: 7 November 2018 / Revised: 6 May 2019 / Accepted: 21 May 2019 / Published online: 23 June 2019

(c) The Author(s), under exclusive licence to European Society of Human Genetics 2019

\begin{abstract}
Incontinentia pigmenti (IP; OMIM\#308300) is a rare genetic disease resulting in neuroectodermal defects, which can lead to disability. At present, there is neither definitive cure available nor are there any sufficiently reliable insights to predict the severity of the disease. We launched the Incontinentia Pigmenti Genetic Biobank (IPGB) project (http://www.igb.cnr.it/ipgb) in 2015 to establish a large-scale deposit of biological samples, to provide detailed clinical information about children diagnosed with IP and to facilitate research. We have built a cohort comprising samples of 381 clinically confirmed patients with IP and 633 healthy individuals recruited through IP patients' associations. The collection includes 269 trios, 83 duos, and 95 families with at least two affected members and represents an extensive dataset (200 cooperative medical institutes, 139 in Italy and 61 worldwide) that enables a comprehensive phenotyping. Joining the IPGB guarantees all participants access to the results including the genetic testing of IP and the long-term storage of the samples. The IPGB is the largest IP sample collection and one of the largest rare-disease-oriented collections in the world and will be open to requests for access to data by the national and international scientific community.
\end{abstract}

\section{Introduction}

Incontinentia pigmenti (IP; OMIM\#308300) is a rare multisystemic genomic disorder [1] with an estimated prevalence at birth of 1.2/100,000 [2]. IP is X-linked dominant, usually lethal in males, and affects the skin and other neuroectodermal tissues in females [3]. IP is caused by variants of the inhibitor of the $K$ polypeptide gene enhancer in $B$ cells, the kinase gamma (IKBKG)/nuclear factor $\kappa B$ $(N F-\kappa B)$ and the essential modulator (NEMO) gene. The $I K B K G$ encoded protein NEMO/IKK $\gamma$ is essential for the NF- $\mathrm{KB}$ activation pathway, involved in a variety of physiological and cellular processes, such as immunity,

Supplementary information The online version of this article (https:// doi.org/10.1038/s41431-019-0451-0) contains supplementary material, which is available to authorized users.

Matilde Valeria Ursini

matildevaleria.ursini@igb.cnr.it

1 Institute of Genetics and Biophysics "A. Buzzati Traverso" CNR, 80131 Naples, Italy

2 University of Basilicata, 85100 Potenza, Italy inflammation, and cell proliferation/survival. Hemizygous $I K B K G / N E M O$ loss of function variants are lethal in males, while IKBKG/NEMO hypomorphic variants, reducing but not abolishing NF- $\mathrm{KB}$ activation, have been identified in male patients with anhidrotic ectodermal dysplasia with immuno deficiency (EDA-ID, OMIM\#300291) and in heterozygous female with IP [4]. Instead, amorphic variants, abolishing NF- $\mathrm{KB}$ activation by complete IKBKG/NEMO gene silencing, cause only IP [4]. Although the classic IP phenotype is almost entirely restricted to females, occasionally 46,XY males present an IP phenotype [5]. These rare cases show the characteristic skin lesions and, in accordance with the pathogenic model of the disease, they are postzygotic genetic mosaics for the IKBKG/NEMO mutation [6-8]. IP has also been diagnosed in males with a 47,XXY karyotype (Klinefelter syndrome) [9].

Despite significant advances in research, the IP/EDA-ID etiology and pathophysiology remains elusive. The rarity of such IP/EDA-ID diseases and their heterogeneous phenotypic manifestation makes it difficult to study their molecular and genetic origin as well as rendering prevention and treatment problematic. More than $30 \%$ of individuals diagnosed with IP were affected by neonatal epilepsy 
leading to a severe form of the disease $[1,10]$. Until now, the absence of any genetic/phenotypic biomarkers able to predict the onset of severe forms makes such IP newborn babies vulnerable to ischemia and other risk factors.

We constituted the biobank with the aim to build a standardized collection of samples and information able to provide biological resources to support and accelerate biomedical research, clinical trials, and the evaluation of early biomarkers. Due to the nature of rare diseases, international cooperation is critical for the sharing of limited numbers of patient samples and the achievement of a critical mass. The rarity of these conditions means that the establishment of a nationwide research platform is vital to facilitate studies on pathogenesis and treatment. In addition, the establishment and maintenance of a widespread collaboration between the biobank and physicians has benefited from the contribution of rare disease patients' organizations. We have developed a strategy, in agreement with the three associations of patients affected by IP, based on genetic information provided upon informed consent and the long-term preservation of patient and family samples.

This paper provides an overview of the IP Genetic Biobank (IPGB) project, launched in 2015, and a profile of the participants who were registered in the first 3 years of the project. The IPGB collects, stores, and distributes valuable human samples and data to improve research on IP.

\section{Methods}

\section{IPGB: definition of the database and of procedures}

The IPGB involved a process (Fig. 1) with specific standard operating procedures (SOPs) that encompass.

\section{Sample and data recruitment}

Patients and their families were interviewed by staff members of the referral hospital, with the IPGB team providing permanent assistance and support (by email and phone: incontientia.pigmenti@igb.cnr.it; 00396132302). All materials used for the study, including documents and the interview guide for the focus group development, were approved by the University of Naples Federico II Ethics Committee Prof. Claudio Buccelli and by the Biobanking and Biomolecular Resources Research Infrastructure- European Research Infrastructure Consortium (BBMRI-ERIC) Ethics Committee. The patients received full information regarding the project from experts and signed specific consent forms. The consent form included an option to withdraw from the study at any time and without giving any explanation. Information and consent forms were provided in different languages for international participants who do not speak English (Step1 and Step2, Fig. 1).

\section{Sample and data collection and storage}

The Ethics Committee specified that blood to be used for scientific purposes might only be taken from under-age patients within the scope of a medical examination. Varying amounts of peripheral blood were collected, $10 \mathrm{ml}$ from adults, $9 \mathrm{ml}$ from children and young people (aged 3-17), 5 $\mathrm{ml}$ from children aged $1-3$, and $2.5 \mathrm{ml}$ from children below 1 year of age. By Ficoll-density centrifugation of $5 \mathrm{ml}$ of blood (for adults), peripheral blood mononuclear cells and granulocytes were obtained and were stored at $-80^{\circ} \mathrm{C}$. The remaining $5 \mathrm{ml}$ of blood was processed to extract the DNA by using the conventional salt precipitation technique (Fig. 2) [11, 12]. Fibroblast cell lines from skin biopsies [13] were generated, when possible, and stored in liquid nitrogen.

The data included socio-demographic variables (sex, date of birth, and nationality), recorded in the IP questionnaire, which were stored in the File Maker Pro Advanced Database. The IPGB project applied a central patient and sample restricted management with unique alpha-numeric code identifiers (IDs) that were used to indicate the study participants and their study data [14] (Step3, Fig.1). The personal and genetic data in the IPGB are processed in line with general data protection regulation.

\section{Sample and data processing: molecular IP diagnosis}

The clinical data and molecular diagnosis results for the IKBKG/NEMO alteration were integrated, by way of a wellstandardized protocol [15-17]. The IP genetic test on the DNA was performed to define the genetic profile of each IP patient. This result confirmed the clinical diagnosis (Step4, Fig. 1).

\section{Sample and data distribution: access criteria}

The collected samples and clinical data can be requested by researchers carrying out studies upon approval by the IPGB access committee. The samples and clinical data are distributed under a strict governance framework, with completion of an application form and upon signature of an institutional material transfer agreement (Step5, Fig.1).

\section{The IPGB and its association with patients' groups}

The IPGB (www.igb.cnr.it/ipgb) was founded in 2015 and obtained funding from patients' associations (IP ASSociation of Italian patients, Onlus, http://www.incontinentia pigmenti.it/, and the IP International Foundation, IPIF, 


\section{IPGB PROCEDURES}

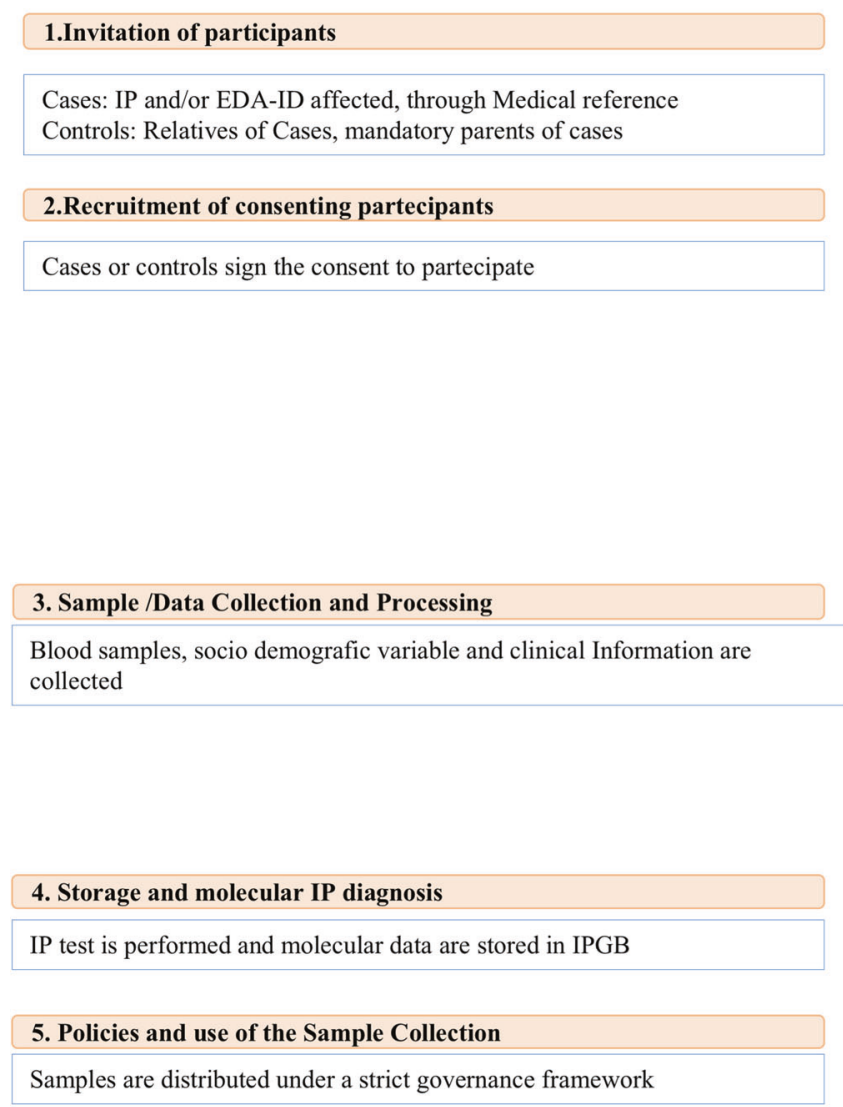

Fig. 1 IPGB Biobank procedures

http://www.ipif.org). The partnerships established with IP patients' associations (IPASSI, Onlus, and IPIF, respectively) and inputs obtained from professionals in human datasets, law, and ethics guided our proposal and sustained the project. To enhance biomedical studies in this area, we conducted a participatory study to assess the feasibility of establishing a cost-effective disease-specific biobank. This disease-specific biobank was conceived and is now administered by researchers at the Institute of Genetics and Biophysics "A. Buzzati-Traverso" CNR (IGB-ABT CNR) and at the Potenza University; the infrastructure for biobanking, responsible for processing, aliquoting, and storing the samples, is located at IGB-ABT CNR. The IPGB has formalized a partnership with the patients' organizations with a written approval signed by the IP association representative of the IPGB project submitted by the IPGB staff. In addition, the IPGB founders have maintained a collaborative scientific partnership since 2009 with the France Incontinentia Pigmenti Association (FIP, http:// incontinentia-pigmenti.fr/) that is not directly involved in

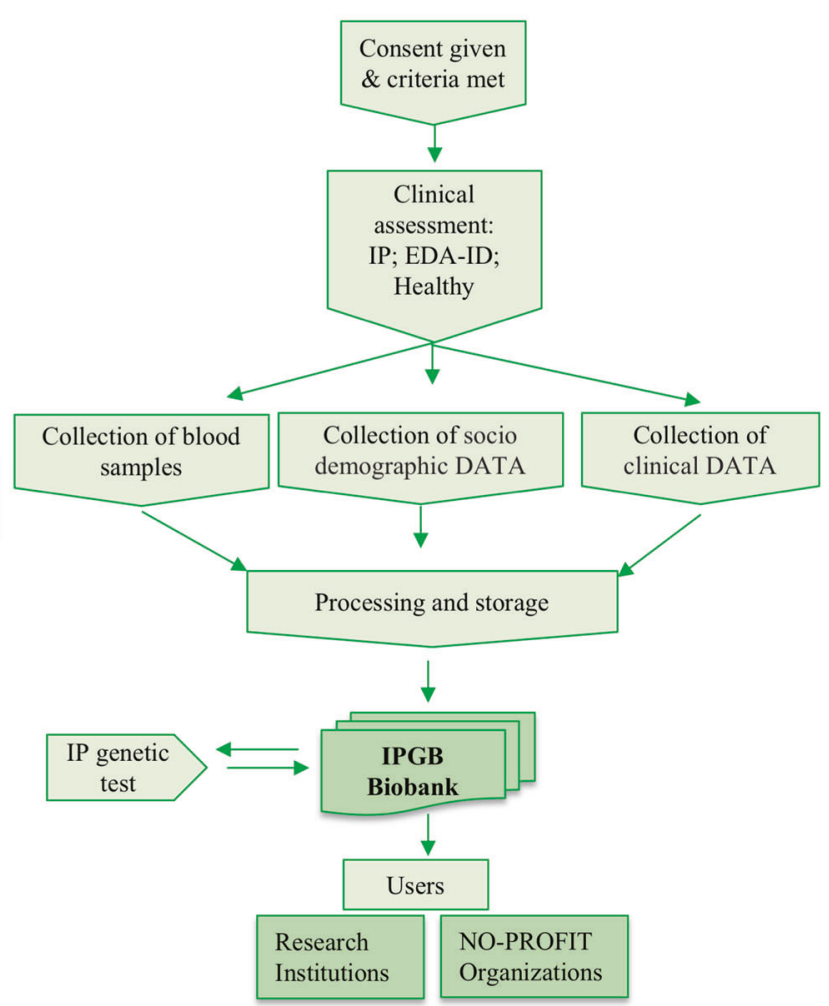

the IPGB project activity but supports our research programs. The agreement between the founders of IPGB and the IPIF dated back to early 2000s, while that with IPASSI Onlus started in 2011 when the association was founded. In 2016, the IPGB Biobank was recognized as an official member of the BBMRI-ERIC (https://www.bbmri.it/home).

\section{Results}

\section{IPGB: current state of the collection}

The IPGB collects biomaterials and data from patients and family members with a confirmed or suspected diagnosis of IP or EDA-ID, NEMO/IKBKG variants being able to cause both diseases [4]. The majority of the sample submissions (63\%) came from local services and from studies carried out in Italy, for reasons of proximity: of the 247 cases from Italy, 205 belong to the IP Historical Collection (IPHC) and 42 to the recent IPGB collection (Table 1). Until now all the 
Fig. 2 Details of the sample processing procedures and blood derivative outputs

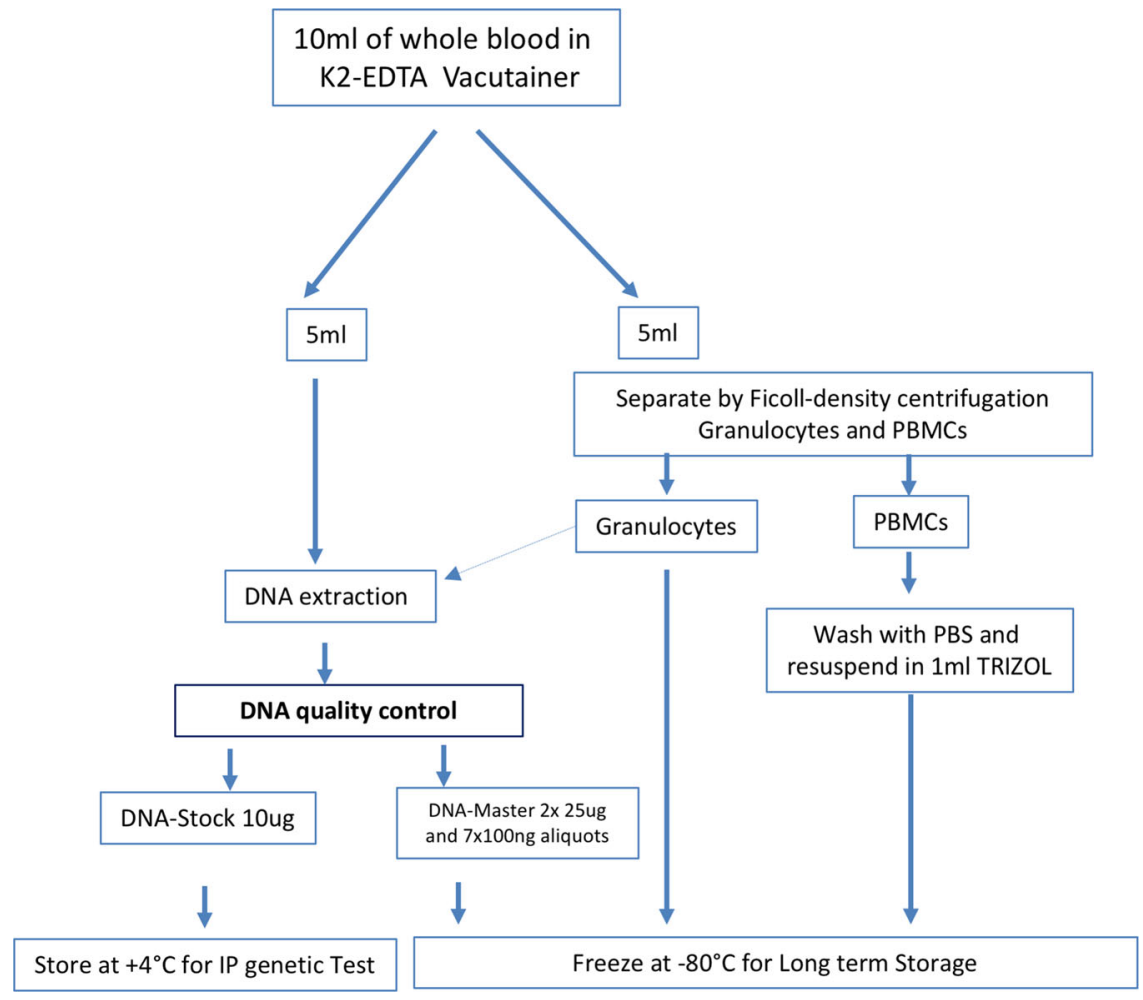

data and samples have been collected from 200 hospitals, representing 139 cooperating medical institutions located throughout Italy and 61 international structures worldwide (Table 1; Fig. 3). The future inclusion of additional hospitals/centers is scheduled. Any possible participation depends on acceptance to implement all the required workflows (Fig. 1) and the participating institutions must confirm the acceptance of the IPGB policies and of the SOPs, realized through a collaboration agreement.

Overall, the collection (the IPHC plus the new IPGB collection) is composed of 388 cases from worldwide of which mostly are IP-female index cases (342 cases, Fig. 4a), 39 samples are from IP mosaic males (Fig. 4b), and 7 from EDA-ID males (Fig. 4c; Table 2). The IPHC consists 334 cases collected since 2000 to 2015 and the new IPGB consists of 54 cases collected from 2015, the year of the foundation of the biobank, until March 2018 (Table 1). With regard to the historical collection (IPHC), it was built for research purpose by the same researchers now operating at the IPGB. Currently, the IPGB and the patients' organization (IPASSI Onlus) are collaborating to contact the source subjects, in order to obtain new consents for the IPGB project. So far, 63 IP patients from the historical collection have been contacted and, in these cases, requests for informed consent have been submitted. The whole collection (including the IPHC and the new IPGB founded in 2015, hereafter named IPGB) is a genetic biobank and preserves parental information in association with the samples. Indeed, it collects samples from index cases and their parents in a structured pedigree: trios, namely the father, mother, and the index case; and duos, namely the mother and the index case. Where possible, both biological parents and other relatives have been invited to participate to obtain complete family trios (or duos). In March 2018, the IPGB collection included 243 trios and 81 duos with female IP-index cases, 22 trios, and 2 duos with IP-male index cases and 4 trios with EDA-ID index cases (Table 3). Samples from the maternal grandparents of 32 IP females are also available (Fig. 4a). There are 55 samples from nonaffected donors (47 sisters and 8 brothers of IP index cases), and 324 samples from mothers (88 IP affected) and 243 from fathers (2 IP-males) (Fig. 4a). For the IP-male index cases, samples from 22 fathers, 24 mothers, and 2 sisters have been collected (Fig. 4b) as healthy relatives. Finally, 3 mothers and 4 fathers are healthy parents of 4 EDA-ID male index cases, and one mother of EDA-ID is IP affected (Fig. 4c, Table 4).

Due to the competence of the founders of the IPGB and the likely high contribution of genetic factors to the phenotypes of interest, the IPGB was mainly conceived as a genetic and data resource. We collected 1326 DNA samples (1132 from a unique withdrawal of blood and 194 from repeated withdrawal of blood of the same donor) extracted from peripheral blood of participants at the cooperating hospitals (Figs. 3 and 4). From 832 donors we have received DNA extracted from blood. From 494 donors we have received peripheral blood samples: for 241/494 we also obtained granulocytes preparation and for $269 / 494$ 
Table 1 Geographical distribution of index cases collection

\begin{tabular}{|c|c|c|c|c|}
\hline Countries & $\begin{array}{l}\text { Case } \\
\text { collection }\end{array}$ & $\begin{array}{l}\text { IPHC } \\
\text { collection }\end{array}$ & $\begin{array}{l}\text { IPGB } \\
\text { collection }\end{array}$ & $\begin{array}{l}\text { Cooperative } \\
\text { medical } \\
\text { institutions }\end{array}$ \\
\hline Australia & 4 & 3 & 1 & 4 \\
\hline Brasile & 1 & 1 & 0 & 1 \\
\hline Bulgaria & 1 & 0 & 1 & 1 \\
\hline Croazia & 2 & 2 & 0 & 2 \\
\hline $\begin{array}{l}\text { Czech } \\
\text { Republic }\end{array}$ & 3 & 3 & 0 & 1 \\
\hline Denmark & 2 & 2 & 0 & 1 \\
\hline Finland & 1 & 1 & 0 & 1 \\
\hline France & 20 & 20 & 0 & 2 \\
\hline Greece & 1 & 1 & 0 & 1 \\
\hline Japan & 1 & 1 & 0 & 1 \\
\hline India & 3 & 2 & 1 & 3 \\
\hline Ireland & 8 & 8 & 0 & 2 \\
\hline Italy & 247 & 205 & 42 & 139 \\
\hline Malaysia & 1 & 1 & 0 & 1 \\
\hline Mexico & 2 & 1 & 1 & 2 \\
\hline Perù & 2 & 1 & 1 & 2 \\
\hline Poland & 4 & 4 & 0 & 3 \\
\hline Serbia & 4 & 4 & 0 & 1 \\
\hline Singapore & 1 & 1 & 0 & 1 \\
\hline Spain & 42 & 40 & 2 & 7 \\
\hline Srilanka & 1 & 1 & 0 & 1 \\
\hline Sweden & 1 & 1 & 0 & 1 \\
\hline Turkey & 10 & 8 & 2 & 8 \\
\hline UK & 22 & 22 & 0 & 12 \\
\hline USA & 1 & 1 & 0 & 1 \\
\hline Venezuela & 3 & 0 & 3 & 1 \\
\hline Total & 388 & 334 & 54 & 200 \\
\hline
\end{tabular}

IPHC incontinentia pigmenti historical collection

we obtained lymphocytes (Table S1). We have collected six skin biopsies: three from IP-female index cases, two from IP-male index cases, and one from an IP female mother. Fibroblast cell lines from skin biopsies of affected tissue of five index case participants (four IP females and one IP male) are stored in liquid nitrogen. The DNA genomic extraction, performed by using the standard method $[11,12]$, produced on average yields of $114.09 \mu \mathrm{g} \pm 81.29$ DNA with, in terms of quality, absorbance ratios of 260/ $280 \mathrm{~nm}$ and 260/230 nm (means $1.89 \pm 0.35$ and $1.81 \pm$ 0.39 , respectively) on 179 samples.

\section{Clinical and genetic profiles of the participants}

We collected baseline clinical information through interviews and reviews of medical records. All the clinical data were obtained for each patient through their completion of a clinical IP questionnaire developed by the IPIF, further extended by the FIP, and by the IPASSI, and validated by the three IP association scientific advisors. The common items addressed during each interview included all phenotypic aspects of IP, and the patient's and her/his family history of the diseases. All the surveys were performed by medical coordinators at the cooperating hospital. Clinical data for each affected family members were obtained via medical reports (doctor's letters or a clinical questionnaire) that were provided by the physicians (IPGB reference) or by the patients themselves. Research staff attempted to follow up any missing data through phone calls or e-mails to the patient's families. For families with multiple affected individuals, clinical information was collected for all affected members. The standardization and comprehensiveness of the clinical data collected allows the classification and stratification of the participants with IP/EDA-ID according to clinical phenotype.

The IPGB is nationally recognized as an expertise center for IP molecular diagnosis. We diagnose all the participants for any genetic alteration in the IP locus upon the acquisition of DNA from affected, under-age donors and nonaffected siblings in addition to medical examinations. As reported in [1], the genetic and clinical profiles of our IP collection revealed heterogeneity of the IP-associated genotypes and phenotypes. About $30 \%$ of the IP index cases (117/388 cases) showed a severe form of IP, involving central nervous system defects (neonatal seizures, ischemic stroke, white matter alterations, etc.), $19.6 \%$ of which (23/ $388,5.9 \%$ cases) had inherited the IKBKG/NEMO mutation from an IP mother with a mild phenotype (intrafamilial heterogeneity). These data are collected by the IPGB to perform genotype-phenotype correlation studies that are at present still not applicable in IP. In addition, we registered socio-demographic variables and clinical and genetic detections.

Because IP is an X-linked dominant disease, lethal in males [3] who can survive only when genetic mosaicism for the NEMO/IKBKG mutation [6] or hypomorphic NEMO/ $I K B K G$ variants are present [4], the male-to-female ratio of the affected cases was very low. We calculated $13.4 \%$ are males and $86.5 \%$ are females. The IP index cases were collected at a mean age of 8.72 years and mode age of 0.083 years (29.88 days from birth), suggesting that the clinical diagnosis is often made very early during infancy (Table S2). We calculated the relative frequency of the age distribution of the appearance of the first dermatological sign corresponding to the clinical diagnosis of IP. The age of onset of the first sign of disease is registered in the clinical questionnaire or, in adults we asked when they had the Stage I/II skin lesions. We observed that in $61 \%$ of cases this occurred between birth and 5 years of age $(0<x<5$ years) (Table S3), and in $45.8 \%$ of cases within the first year 


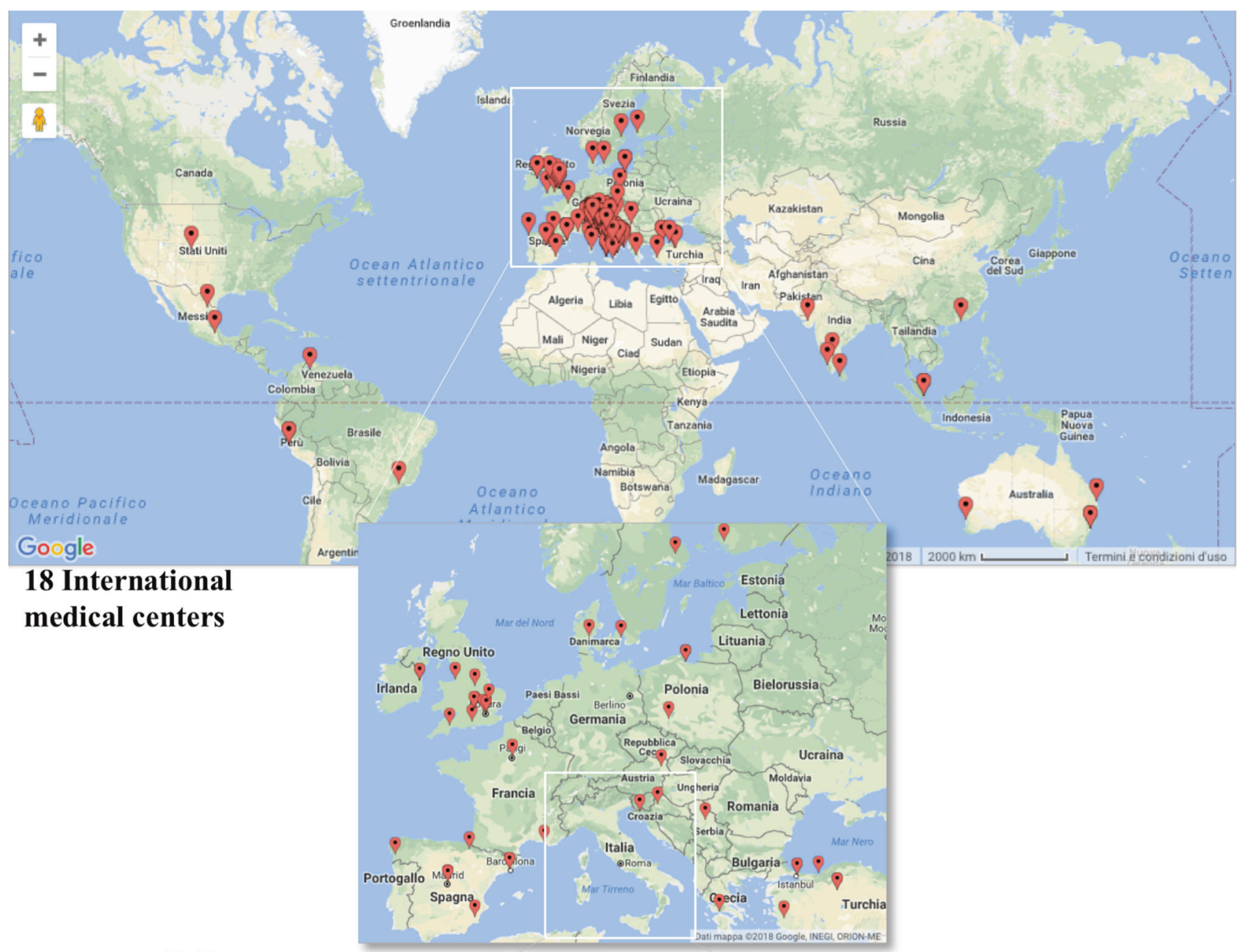

\section{European medical} centers

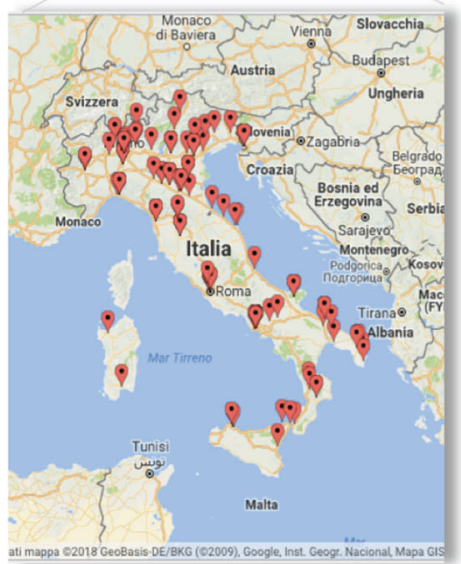

\section{9 italian medical centers}

Fig. 3 Geographical distribution of the cooperating hospitals

(Table S4), often between the first and second month of life (20.7\%) (Table S5). Currently, the patients' age range from 1 to 58 years, and about the $59 \%$ of them are between 5 and 20 years. The analysis of the IP-male index cases showed that the corresponding values were 5.83 years old (mean age) and 1.58 years (mode age) (31 cases available); for the EDA-ID males the age of clinical diagnosis was 2.19 years (Table S2). To analyze the burden of the IP disease on the 
A

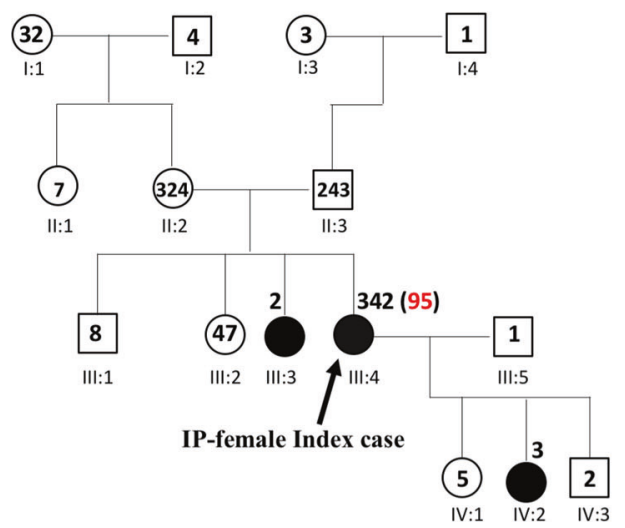

Fig. 4 Collection of IP (a), IP-male (b), and EDA-ID (c) index cases with relatives. In the figure the distribution of donors is shown in relation to the Index cases. The number of cases is indicated inside or near the pedigree symbol where possible. The number of repeated withdrawals is shown in red. All index cases (females and males, IP or
B
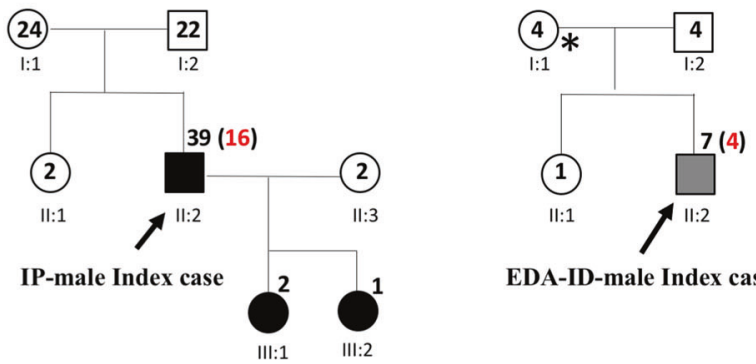

EDA-ID-male Index case

EDA-ID) have deposited the DNA sample and a completed IP clinical questionnaire. For all relatives, we collected the DNA sample and only when they were affected we collected the clinical questionnaire. The arrows indicated index case. Asterisk: The mothers of EDA-ID are three healthy and one IP affected
Table 2 Spectrum of the disease groups

\begin{tabular}{lllll}
\hline Disease groups & Case collection & IPHC collection & IPGB pending & IPGB collection \\
\hline IP-female index cases & 342 & 233 & 63 & 46 \\
IP-male index cases & 39 & 33 & 0 & 6 \\
EDA-ID male index cases & 7 & 5 & 0 & 2 \\
Total & 388 & 271 & 63 & 54 \\
\hline
\end{tabular}

To be required for the permission for depositing in IPGB

Table 3 Spectrum of the genealogical data of each index case

\begin{tabular}{lllll}
\hline Disease group & Case collection & DUOS (mother and index case) & TRIOS (parents and index case) & SINGLETON (only index case) \\
\hline IP-female index cases & 342 & 81 & 243 & 18 \\
IP-male index cases & 39 & 2 & 22 & 15 \\
EDA-ID male index cases & 7 & 0 & 4 & 3 \\
Total & 388 & $83(61)^{\mathrm{a}}$ & $269(178)^{\mathrm{a}}$ & 36 \\
\hline
\end{tabular}

${ }^{a}$ Number of the index cases in simplex versus multiplex families

Italian population, we calculated the birth prevalence of IP female affected subjects from 2002 to 2017 (Table S6) and we observed that the mean birth prevalence is $1.6 \times 10^{-5}$, higher than the value of $1.2 \times 10^{-5}$ reported in [2] as the estimated incidence of European cases. We noticed that the percentage of sporadic cases is $70 \%$.

\section{Discussion}

In this paper, we have described the largest IP cohort in the world in terms of data and biological samples collection, the IPGB. To the best of our knowledge the IPGB is one of the largest rare-disease-oriented collections. All information collected in the IPGB is precious for the following reasons:
1. The IPGB collects trios (in some cases multiple generations) in order to preserve the genetic and phenotypic association between biological samples;

2. The IPGB produces and collects IP molecular test results, thus meaning that the samples and data of IP index cases can be harmonized for IP locus alterations;

3. The IPGB collects clinical data, updated over time, enabling genotype-phenotype studies;

4. The IPGB collects socio-demographic data, facilitating epidemiological study.

As an example, we observed that the median age of appearance of the first IP dermatological sign is at day 29.88 from birth that represents an important finding in terms of the clinical diagnosis of IP. Such analysis 
Table 4 Distribution of phenotype of relatives

\begin{tabular}{|c|c|c|c|c|}
\hline & Index cases & $\begin{array}{l}\text { Affected } \\
\text { relatives }\end{array}$ & $\begin{array}{l}\text { Healthy } \\
\text { relatives }\end{array}$ & Total \\
\hline IP-female index cases & 342 & & & 342 \\
\hline Mother & & 88 & 236 & 324 \\
\hline Father & & 0 & 243 & 243 \\
\hline Sister & & 2 & 47 & 49 \\
\hline Brother & & 0 & 8 & 8 \\
\hline Child of IP-index case & & 3 & 7 & 10 \\
\hline Maternal grandmother & & 3 & 29 & 32 \\
\hline Maternal grandfather & & 0 & 4 & 4 \\
\hline Paternalgrandmother & & 0 & 3 & 3 \\
\hline Paternal grandfather & & 0 & 1 & 1 \\
\hline Aunts & & 0 & 7 & 7 \\
\hline IP-male index cases & 39 & & & 39 \\
\hline $\begin{array}{l}\text { Child of IP male- } \\
\text { index case }\end{array}$ & & 3 & 0 & 3 \\
\hline Mother & & 0 & 24 & 24 \\
\hline Father & & 0 & 22 & 22 \\
\hline Sister & & 0 & 2 & 2 \\
\hline IP phenotype total & 381 & 99 & 633 & 1113 \\
\hline $\begin{array}{l}\text { IP cases husband and } \\
\text { wife }\end{array}$ & & 0 & 3 & 3 \\
\hline $\begin{array}{l}\text { EDA-ID male index } \\
\text { cases }\end{array}$ & 7 & & & 7 \\
\hline Mother & & 1 & 3 & 4 \\
\hline Father & & 0 & 4 & 4 \\
\hline Sister & & 0 & 1 & 1 \\
\hline $\begin{array}{l}\text { EDA-ID phenotype } \\
\text { total }\end{array}$ & 7 & 1 & 8 & 16 \\
\hline
\end{tabular}

extended to the other relevant signs of IP could be to set up a clinical follow-up protocol for IP patients to avoid psychological stress in the management of the disease. To realize this study of natural history of IP disease is essential the involvement of patients' organizations because they raise awareness, interest, and trust in biobanks. This has been instrumental in gaining the critical mass of samples essential for research into this rare disease. The IPGB is allied with three patients' associations, two of which are directly involved in the enrolment of participants and in raising funds for the biobank project (IPASSI and IPIF), with the third serving as an advisor and collaborator, fostering the dissemination of information about the IPGB and helping to fund the collateral research activity of IPGB staff members.

The role of the IPGB is not only to collect samples and data from patients with IP but also to use them for research purposes. The IPGB enables scientists in different countries to propose interdisciplinary collaborations on common research projects based on the complementary strengths and sharing of skills, with a clear benefit for patients. The IPGB aims to reduce the time period required for the recruitment of appropriate samples and data, a very complex phase especially in the case of rare diseases. The standardized collection of samples and information of the IPGB can be used in transnational research proposals to accelerate diagnosis or in omic or multiomic integrated approaches for the discovery of genetic and/or phenotypic biomarkers to predict the onset of severe forms of IP and for the development of personalized therapy. Access to the IPGB can be requested by submitting a research application to the IPGB Scientific Board, which consists of experts in the fields of IP, in epidemiology, human genetics, basic research, ethics, and health care law. The use and access regulations and policies comprise (1) general rules for cooperative studies, (2) forms and templates to submit the research proposals, (3) a well-defined process of decision, and (4) publication guidelines. The sample donors have transferred the right to use their biomaterials for scientific purposes to the IPGB, which reserves the right to use such samples until the time the sample donors should withdraw their consent.

In conclusion, a fundamental prerequisite for the construction of a valuable collection of samples was the agreement with patients' associations, which illustrated the operating rules to the patients and their families giving them the opportunity to express opinions, needs, and concerns. In addition, the IP patients' organizations have kept the associated families and referring clinicians informed of the IPGB's activities and policies, promoted the recruitment of patients and relatives, and raised funds to support the IPGB in order to improve research on rare disease.

\section{Access to the IPGB}

A research application to the IPGB Scientific Board, which includes a representative of the IP patients' organizations, has to be submitted. The final decision is made by the IPGB Steering Committee and by the representatives of the institution where the samples have been collected. Decision criteria are the project's feasibility, the clear indication of well-defined objectives and benefit for patients, the inclusion of the applicant within the IP research community, and a stringent scheduling. Appropriate expertise in the field of interest is therefore a prerequisite. Upon approval, collaborating research groups and institutions must agree to make their data available to research community for future studies upon completion and publication of their study.

Academic and noncommercial researchers are eligible to apply and to use samples and/or anonymized data. 
Application and review procedures are in place. Researchers should present a sound scientific rationale for the proposed study, have a good research track record, and be supported by their institution. The following types of studies will be prioritized: studies testing or generating new hypotheses on the pathophysiology of IP/EDA-ID; studies improving diagnosis and phenotyping; and basic science studies, e.g., pharmacological in vitro studies, potentially leading to clinical trials of therapeutic approaches.

The release of data and/or samples will be enabled upon the signing of a materials transfer agreement and/or data transfer agreement, and any such researchers must not attempt to identify any individuals.

The IPGB has been planned to operate on a cost recovery basis to replace any released samples, thereby helping to ensure the long-term sustainability of the resource.

Additional information and application forms can be found on the Biobank website: http://ipgb.cnr.it.

\section{Sustainability}

Since January 2015 the IPGB was supported by the IPASSI and IPIF.

\section{Bioresource location}

The project is led and managed by the IP team, located within the IGB-ABT CNR. The participants' data are securely stored at the IPGB database in locked files and in a bespoke database. Deidentified biological samples are stored at the IPGB Biobank.

Bioresource contact: incontinentia.pigmenti@igb.cnr.it

Bioresource URL http://www.igb.cnr.it/ipgb

The IPGB Biobank is an IP project and, therefore, the bioresource's URL is linked to the IP group webpage. Researchers are encouraged to seek information through this site and/or contact the IP team.

Acknowledgements We would like to thank the patients and their families and physicians. We would also like to thank the Italian Incontinentia Pigmenti Association (www.incontinentiapigmenti.it), the French Incontinentia Pigmenti Association (http://incontinentia pigmenti.fr), and the IPIF (www.ipif.org).

Funding All phases of this study have been supported by the IPIF (http://www.ipif.org), the Italian Incontinentia Pigmenti Association (IPASSI, http://www.incontinentiapigmenti.it), the FIP (http:// incontinentia-pigmenti.fr), by CNR-DSB Progetto Bandiera "InterOmics" and by PON FERS \#F/050011/02/X32 "Functional genomics of rare genetic diseases: Creation of innovative tools with high diagnostic power" to Drs Fusco and Ursini.

Author contributions Conceived and designed the research study: FF, MBL, and MVU. Conducted the collection: VV and DF. Analyzed the data: FF and MVU. Wrote the manuscript: FF, AP, and MVU.

\section{Compliance with ethical standards}

Conflict of interest The authors declare that they have no conflict of interest.

Publisher's note: Springer Nature remains neutral with regard to jurisdictional claims in published maps and institutional affiliations.

\section{References}

1. Fusco F, Paciolla M, Conte MI, Pescatore A, Esposito E, Mirabelli P, et al. Incontinentia pigmenti: report on data from 2000 to 2013. Orphanet J Rare Dis. 2014;9:93.

2. Orphanet Report Series-Prevalence of rare diseases: bibliographic data-June 2018-Number. https://www.orpha.net/orpha com/cahiers/docs/GB/Prevalence_of_rare_diseases_by_alpha betical_list.pdf

3. Scheuerle AE, Ursini MV. Incontinentia pigmenti. In: Adam MP, Ardinger HH, Pagon RA, Wallace SE, Bean LJH, Stephens K, Amemiya A, editors. GeneReviews ${ }^{\circledR}$. Seattle, WA: University of Washington; 1993-2018. 1999 Jun 8 [updated 2017 Dec 21].

4. Fusco F, Pescatore A, Conte MI, Mirabelli P, Paciolla M, Esposito E, et al. EDA-ID and IP, two faces of the same coin: how the same IKBKG/NEMO mutation affecting the NF- $\mathrm{KB}$ pathway can cause immunodeficiency and/or inflammation. Int Rev Immunol. 2015;34:445-59.

5. Scheuerle AE. Male cases of incontinentia pigmenti: case report and review. Am J Med Genet. 1998;77:201-18.

6. Fusco F, Conte MI, Diociaiuti A, Bigoni S, Branda MF, Ferlini A, et al. Unusual father- to-daughter transmission of incontinentia pigmenti due to mosaicism in IP males. Pediatrics. 2017;140: e20162950.

7. Hull S, Arno G, Thomson P, Mutch S, Webster AR, Rai H, et al. Somatic mosaicism of a novel IKBKG mutation in a male patient with incontinentia pigmenti. Am J Med Genet. 2015;167:1601-4.

8. Fusco F, Fimiani G, Tadini G, Michele D, Ursini MV. Clinical diagnosis of incontinentia pigmenti in a cohort of male patients. $\mathrm{J}$ Am Acad Dermatol. 2007;56:264-7.

9. Kenwrick S, Woffendin H, Jakins T, Shuttleworth SG, Mayer E, Greenhalgh L, et al. International IP Consortium. Survival of male patients with incontinentia pigmenti carrying a lethal mutation can be explained by somatic mosaicism or Klinefelter syndrome. Am J Hum Genet. 2001;69:1210-7.

10. Minić S, Trpinac D, Obradović M. Systematic review of central nervous system anomalies in incontinentia pigmenti. Orphanet $\mathbf{J}$ Rare Dis. 2013;8:25.

11. Maniatis T, Fritsch EF, and Sambrook J. Molecular cloning: a laboratory manual. Cold Spring Harbor, N.Y.: Cold Spring Harbor Laboratory; 1982.

12. Miller SA, Dykes DD, Polesky HF. A simple salting out procedure for extracting DNA from human nucleated cells. Nucleic Acids Res. 1988;16:1215.

13. Vangipuram M, Ting D, Kim S, Diaz R, Schüle B. Skin punch biopsy explant culture for derivation of primary human fibroblasts. J Vis Exp. 2013;77:e3779. https://doi.org/10.3791/3779.

14. Olden M, Holle R, Heid IM, Stark K. IDGenerator: unique identifier generator for epidemiologic or clinical studies. BMC Med Res Methodol. 2016;16:120. https://doi.org/10.1186/s12874016-0222-3.

15. Fusco F, Paciolla M, Napolitano F, Pescatore A, D'Addario I, Bal $\mathrm{E}$, et al. Genomic architecture at the incontinentia pigmenti locus favours de novo pathological alleles through different mechanisms. Hum Mol Genet. 2012;21:1260-71. 
16. Fusco F, Bardaro T, Fimiani G, Mercadante V, Miano MG, Falco $\mathrm{G}$, et al. Molecular analysis of the genetic defect in a large cohort of IP patients and identification of novel NEMO mutations interfering with NF-кB activation. Hum Mol Genet. 2004;13: 1763-73.
17. Bardaro T, Falco G, Sparago A, Mercadante V, Gean Molins E, Tarantino E, et al. Two cases of misinterpretation of molecular results in incontinentia pigmenti, and a PCR- based method to discriminate NEMO/IKK $\gamma$ gene deletion. Hum Mutat. 2003; 21:8-11. 